Pacific Journal of Mathematics

TWO THEOREMS OF GAUSS AND ALLIED IDENTITIES 


\title{
TWO THEOREMS OF GAUSS AND ALLIED IDENTITIES PROVED ARITHMETICALLY
}

\author{
GEORGE E. ANDREWS
}

The product formulae of Gauss for the theta functions $\theta_{4}(0, q)$ and $(1 / 2)(-q)^{-1 / 8} \theta_{2}\left(0,(-q)^{1 / 2}\right)$ have been derived in many ways by analytic means. In this paper these formulae are derived by enumerating certain types of partitions. The enumeration technique is shown to be applicable to more general results, and several important theorems in basic hypergeometric series are proved from suitable enumerations of partitions.

In [7], F. Franklin gave his now famous arithmetic proof of Euler's identity:

$$
\prod_{n=1}^{\infty}\left(1-q^{n}\right)=\sum_{n=-\infty}^{\infty}(-1)^{n} q^{(1 / 2) n(3 n-1)} .
$$

As is well-known, identity (1.1) can be deduced from Jacobi's triple product identity [10; p. 284]:

$$
\prod_{n=0}^{\infty}\left(1-q^{2 n+2}\right)\left(1+z q^{2 n+1}\right)\left(1+z^{-1} q^{2 n+1}\right)=\sum_{n=-\infty}^{\infty} q^{n^{2}} z^{n} .
$$

Several arithmetic proofs of (1.2) have appeared (e.g. [14; pp. 34-36], [15; pp. 10-12], [13], [16], and [2; pp. 561-562]). The known arithemetic proofs of (1.2) differ considerably from Franklin's proof of (1.1) probably because of the much greater generality of Jacobi's identity.

In this paper, we begin by considering the following two identities due to Gauss [8; p. 447, eqs. (14) and (10)]:

$$
\begin{aligned}
\prod_{n=1}^{\infty} \frac{\left(1-q^{2 n}\right)}{\left(1+q^{2 n-1}\right)} & =1+\sum_{n=1}^{\infty}(-1)^{n} q^{n(2 n-1)}\left(1+q^{2 n}\right) \\
& =\frac{1}{2} i(-q)^{-1 / 8} \theta_{2}\left(0,(-q)^{1 i^{12}}\right),
\end{aligned}
$$

and

$$
\prod_{n=1}^{\infty} \frac{\left(1-q^{n}\right)}{\left(1+q^{n}\right)}=1+2 \sum_{n=1}^{\infty}(-1)^{n} q^{n^{2}}=\theta_{4}(0, q) .
$$

These identities may be directly deduced from Jacobi's identity if one employs also the further result of Euler [10; p. 277]:

$$
\prod_{n=1}^{\infty}\left(1+q^{n}\right)=\prod_{n=1}^{\infty} \frac{1}{\left(1-q^{2 n-1}\right)} .
$$


Our first object will be to give arithmetic proofs of (1.3) and (1.4); these proofs turn out to be much more closely related to Franklin's technique than to the above-mentioned proofs of Jacobi's identity. In $\S 3$, we shall examine these proofs as well as Franklin's proof of (1.1) in order to deduce two variable series-product identities totally different from Jacobi's identity. Indeed, the series we obtain will all be special cases of the identity:

$$
(1-t) \sum_{n=0}^{\infty} \frac{(a)_{n} t^{n}}{(b)_{n}}=\sum_{n=0}^{\infty} \frac{(a)_{n}(a t q / b)_{n} b^{n} t^{n} q^{n^{2}-n}\left(1-a t q^{2 n}\right)}{(b)_{n}(t q)_{n}}
$$

where

$$
(a ; q)_{n}=(a)_{n}=(1-a)(1-a q) \cdots\left(1-a q^{n-1}\right),
$$

and

$$
(a ; q)_{\infty}=(a)_{\infty}=\lim _{n \rightarrow \infty}(a)_{n}
$$

This identity appears to have been originally discovered by L. J. Rogers [11; p. 334], and independently by N. J. Fine [5; eq. (14.1)]; it is a special case of Watson's $q$-analog of Whipple's theorem [12; p. 100, eq. (3.4.1.5)]. Section 4 will then be devoted to an arithmetic proof of the full Rogers-Fine identity (1.6). Finally in $\S 5$, we shall illustrate the usefulness of the techniques developed in $\S \S 3$ and 4 by proving arithmetically further identities of the basic hypergeometric type.

2. Arithmetic proofs of $(1.3)$ and $(1.4)$. Let $G_{e}(n)$ (resp. $\left.G_{0}(n)\right)$ denote the number of partitions of $n$ into an even (resp. odd) number of parts in which no even parts are repeated. Then clearly

$$
\prod_{n=1}^{\infty} \frac{\left(1-q^{2 n}\right)}{\left(1+q^{2 n-1}\right)}=1+\sum_{n=1}^{\infty}\left(G_{e}(n)-G_{0}(n)\right) q^{n} \text {. }
$$

Hence we may restate (1.3) arithmetically in the following theorem:

THEOREM 1. If $n \geqq 1$, then

$$
G_{e}(n)-G_{0}(n)=\left\{\begin{array}{l}
(-1)^{j} \quad \text { if } n=j(2 j \pm 1), \\
0 \quad \text { otherwise } .
\end{array}\right.
$$

Proof. We shall use (in slightly altered form) the notation of Richard Guy [9], which he introduced in an arithmetic proof of the reciprocal of (1.5).

We call the face $f(\pi)$ of a partition $\pi$ the number of nodes in the two righthandmost columns of the associated Ferrars graph. Thus 
if $\pi$ denotes the partition $7+7+7+6+3+2$, the Ferrars graph is

and thus $f(\pi)=7$.

In exact agreement with Guy [9], we define the base $b(\pi)$ of a partition $\pi$ to be the smallest part appearing; thus for the above partition $b(\pi)=2$. We also denote by $l(\pi)$ the largest part of $\pi$, by $\nu(\pi)$ the number of appearances of the largest part, and by $\#(\pi)$ the total number of parts of $\pi$.

We let $\pi_{G}(n)$ denote the set of all partitions of $n$ in which no even parts are repeated, and we define

$$
\begin{aligned}
A_{1}(n) & =\left\{\pi \mid \pi \in \pi_{G}(n), l(\pi) \text { even }\right\} \\
A_{2}(n) & =\left\{\pi \mid \pi \in \pi_{G}(n), l(\pi) \text { odd, } b(\pi)=1\right\} \\
A_{3}(n) & =\left\{\pi \mid \pi \in \pi_{G}(n), l(\pi) \text { odd, } f(\pi) \text { even, } b(\pi)>f(\pi)\right\} \\
A_{4}(n) & =\left\{\pi \mid \pi \in \pi_{G}(n), l(\pi) \text { odd, } b(\pi) \text { even, } \nu(\pi) \geqq \frac{1}{2} b(\pi)\right\} \\
A_{5}(n) & =\left\{\pi \mid \pi \in \pi_{G}(n), l(\pi) \text { odd, } f(\pi) \text { odd, } f(\pi) \geqq b(\pi)\right\} \\
A_{6}(n) & =\left\{\pi \mid \pi \in \pi_{G}(n), l(\pi) \text { odd, } b(\pi) \text { odd, } b(\pi) \neq 1, \nu(\pi)\right. \\
& \left.\geqq \frac{1}{2}(b(\pi)+1)\right\} .
\end{aligned}
$$

It is a simple matter to verify that

$$
\pi_{G}(n)=\bigcup_{i=1}^{6} A_{i}(n)
$$

is a disjoint set-theoretic partitioning of $\pi_{G}(n)$.

We now define three functions $\alpha, \beta$, and $\gamma$ :

$$
\alpha: A_{1}(n) \rightarrow A_{2}(n),
$$

where for $\pi \in A_{1}(n)$ and $\pi$ of the form $n=b_{1}+b_{2}+\cdots+b_{s}$, with $b_{i} \geqq b_{i+1}, \alpha(\pi)=\pi^{\prime}$ where $\pi^{\prime}$ is of the form

$$
n=\left(b_{1}-1\right)+b_{2}+\cdots+b_{s}+1 \text {. }
$$

$$
\beta: A_{3}(n) \rightarrow A_{4}(n),
$$


where for $\pi \in A_{3}(n)$ and $\pi$ of the form $n=b_{1}+b_{2}+\cdots+b_{s}$ with $b_{i} \geqq b_{i+1}, \beta(\pi)=\pi^{\prime \prime}$ where $\pi^{\prime \prime}$ is of the form (note $\nu(\pi)=(1 / 2) f(\pi)$ )

$$
\begin{gathered}
n=\left(b_{1}-2\right)+\left(b_{2}-2\right)+\cdots+\left(b_{(1 / 2) f(\pi)}-2\right) \\
+b_{1 / 2(f(\pi)+1)}+\cdots+b_{s}+f(\pi) . \\
\gamma: A_{5}(n) \rightarrow A_{6}(n),
\end{gathered}
$$

where for $\pi \in A_{5}(n)$ and $\pi$ of the form $n=b_{1}+b_{2}+\cdots+b_{s}$ with $b_{i} \geqq b_{i+1}, \gamma(\pi)=\pi^{\prime \prime}$, where $\pi^{\prime \prime}$ is of the form (note $\nu(\pi)=1 / 2(f(\pi)-1)$ ).

$$
\begin{aligned}
n= & \left(b_{i}-2\right)+\left(b_{i}-2\right)+\cdots+\left(b_{1 / 2(f(\pi)-1)}-2\right)+\left(b_{1 / 2(f(\pi)+1)}-1\right) \\
& +b_{1 / 2(f(\pi)+3)}+\cdots+b_{s}+f(\pi) .
\end{aligned}
$$

The idea used in the construction of these mappings is similar to that of Franklin [7]. The mapping $\alpha$ shifts a single node in the Ferrars graph from the right hand most position to the bottom most position (and vice-versa by $\alpha^{-1}$ ).

The mapping $\beta$ transposes a face with an even number of nodes into a base (and $\beta^{-1}$ reverses this process), and $\gamma$ transposes a face with an odd number of nodes into a base (and $\gamma^{-1}$ reverses this process). From this point of view, one may easily verify that each of these mappings is one-to-one and onto provided they are defined.

The only cases where the above maps are not defined are for partitions of the form

$$
n=\underbrace{(2 m+1)+(2 m+1)+\cdots+(2 m+1)}_{m \text { terms }},
$$

or

$$
n=\underbrace{(2 m-1)+(2 m-1)+\ldots+(2 m-1)}_{m \text { terms }} .
$$

The first of these partitions is in $A_{3}(n)$; however $\beta(\pi)$ does not exist in this case because transposition of the face to the base produces a situation in which the base exceeds all other parts, an impossibility. The second of these partitions is in $A_{6}(n)$, however $\gamma^{-1}(\pi)$ does not exist in this case because transposition of the base to the face produces a situation in which the bottom node of the new face does not fit onto the remaining nodes, again an impossibility.

Upon inspection we see that each of our three maps pairs partitions $\pi$ and $\pi^{\prime}$ with $\#(\pi) \not \equiv \#\left(\pi^{\prime}\right)(\bmod 2)$. Hence

$$
(-1)^{\sharp(\pi)}+(-1)^{\sharp\left(\pi^{\prime}\right)}=0 \text {. }
$$

Thus apart from the exceptional cases described above,

$$
G_{e}(n)-G_{0}(n)=0
$$


In the exceptional cases, we have an extra contribution, namely

$$
G_{e}(n)-G_{0}(n)=(-1)^{m} \quad \text { if } \quad n=m(2 m \pm 1) .
$$

Thus Theorem 1 is established.

To prove Gauss's second identity (1.4), we let $g_{e}(n)$ (resp. $g_{0}(n)$ ) denote the number of partitions of $n$ of the form

$$
n=\sum_{i=1}^{s} a_{i}+\sum_{j=1}^{t} b_{j}
$$

with $\alpha_{i}>a_{i+1}, b_{j} \geqq b_{j+1}$ and $s+t$ even (resp. odd). As before, we see that

$$
\prod_{n=1}^{\infty} \frac{\left(1-q^{n}\right)}{\left(1+q^{n}\right)}=1+\sum\left(g_{e}(n)-g_{0}(n)\right) q^{n} .
$$

Hence we may restate (1.4) arithmetically in the following theorem.

Theorem 2. If $n \geqq 1$, then

$$
g_{e}(n)-g_{0}(n)=\left\{\begin{array}{l}
2(-1)^{m} \quad \text { if } n=m^{2} \\
0 \quad \text { otherwise }
\end{array}\right.
$$

Proof. We must first introduce some terminology related to partitions $\pi$ of the form

$$
n=\sum_{i=1}^{s} a_{i}+\sum_{j=1}^{t} b_{j}
$$

where $a_{i}>a_{i+1}, b_{j} \geqq b_{j+1}$.

We define the face $F(\pi)$ of such a partition to be the total number of appearances of the largest summand; by $F_{b}(\pi)$, we denote the total number of $b_{i}$ equal to the largest summand. We say the face of $\pi$ is split if $a_{1}=l(\pi)$; otherwise we say the face of $\pi$ is unified. As before, the base $b(\pi)$ is the smallest part in $\pi$. If $b(\pi)$ occurs among the $b_{i}$, we say $\pi$ is $b$-based; otherwise we say $\pi$ is $a$-based. We define $\Delta(n)$ as the set of those partitions of $n$ with $a_{1}=\lambda, b_{1}=b_{2} \cdots=b_{t}=\lambda, s=1$, $t=\lambda$ (thus $\Delta(n)$ is empty if $n$ is not twice a triangular number and is a singleton if $n=2 \lambda(\lambda+1) / 2)$. We let $\pi_{g}(n)$ denote the set of all partitions of $n$ of the type defined above, and we define

$$
\begin{aligned}
& B_{1}(n)=\left\{\pi \mid \pi \in \pi_{g}(n), \text { split face, } F(\pi) \leqq b(\pi)\right\} \\
& B_{2}(n)=\left\{\pi \mid \pi \in \pi_{g}(n), b \text {-based, } b(\pi) \leqq F_{b}(\pi)\right\}-\Delta(n) \\
& B_{3}(n)=\left\{\pi \mid \pi \in \pi_{g}(n), \text { unified face, } F(\pi)<b(\pi)\right\} \\
& B_{4}(n)=\left\{\pi \mid \pi \in \pi_{g}(n), \text { a-based, } b(\pi)<F(\pi)\right\} \cup \Delta(n) .
\end{aligned}
$$

We now define two functions. 


$$
\delta: B_{1}(n) \rightarrow B_{2}(n)
$$

where if $\pi \in B_{1}(n)$ and is given by $n=a_{1}+a_{2}+\cdots+a_{s}+b_{1}+b_{2}+\cdots+b_{t}$, then $\delta(\pi)$ is given by

$$
\begin{gathered}
n=\underbrace{a_{2}+\cdots+a_{s}}_{\text {new } a \text {-component }} \\
+\underbrace{\left(a_{1}-1\right)+\left(b_{1}-1\right)+\cdots+\left(b_{F(\pi)-1}-1\right)+b_{F(\pi)}+\cdots+b_{t}+F(\pi)}_{\text {new b-component }} . \\
\zeta: B_{3}(n) \rightarrow B_{4}(n)
\end{gathered}
$$

where if $\pi \in B_{3}(n)$ and is given by $n=a_{1}+a_{2}+\cdots+a_{s}+b_{1}+b_{2}+\cdots+b_{t}$, $\zeta(\pi)$ is given by

$$
n=\underbrace{a_{1}+a_{2}+\cdots+a_{s}+F(\pi)}_{\text {new } a \text {-component }}+\underbrace{\left(b_{1}-1\right)+\cdots+\left(b_{F(\pi)}-1\right)+b_{2}+\cdots+b_{t}}_{\text {new } b \text {-component }} .
$$

The idea behind these mappings resembles that in Theorem 1 . The mapping $\delta$ takes the split face of a partition and inserts it as a minimal term in the $b$-component while shifting the largest remaining $a$-term over to the $b$-component and vice-versa with $\delta^{-1}$. The mapping $\xi$ takes the unified face of a partition and inserts it as a minimal term in the $a$-component (while $\xi^{-1}$ reverses this process).

It is easily verified that

$$
\pi_{g}(n)=\bigcup_{j=1}^{4} B_{j}(n)
$$

is a disjoint set-theoretic partitioning of $\pi_{g}(n)$. Also the mappings $\delta$ and $\xi$ are clearly one-to-one and onto provided they are defined. The only cases where the maps or their inverses are undefined are

$$
s=1, t=m-1, a_{1}=b_{1}=\cdots=b_{t}=m
$$

and

$$
s=0, t=m, b_{1}=\cdots=b_{t}=m .
$$

Upon inspection we see that both $\delta$ and $\xi$ pair partitions $\pi$ and $\pi^{\prime}$ in $\pi_{g}(n)$ with $\sharp(\pi) \not \equiv \#\left(\pi^{\prime}\right)(\bmod 2)$; hence for such pairs

$$
(-1)^{\sharp(\pi)}+(-)^{\sharp\left(\pi^{\prime}\right)}=0 \text {. }
$$

Thus we see that apart from the exceptional cases described above

$$
g_{e}(n)-g_{0}(n)=0 \text {. }
$$

In the exceptional cases, we have two extra contributions, and so

$$
g_{e}(n)-g_{0}(n)=2(-1)^{m} \text { if } n=m^{2} .
$$


This concludes the proof of Theorem 2 .

To make clear these proofs we give examples of the mappings utilized in Theorems 1 and 2. In Theorem 1, with $n=7$

$$
\begin{gathered}
7 \stackrel{\beta}{\longrightarrow} 5+2 \\
6+1 \stackrel{\alpha}{\longrightarrow} 5+1+1 \\
4+3 \stackrel{\alpha}{\longrightarrow} 3+3+1 \\
4+2+1 \stackrel{\alpha}{\longrightarrow} 3+2+1+1 \\
4+1+1+1 \stackrel{\alpha}{\longrightarrow} 3+1+1+1+1 \\
2+1+1+1+1+1 \stackrel{\alpha}{\longrightarrow} 1+1+1+1+1+1+1 .
\end{gathered}
$$

In Theorem 2, with $n=4$ (below we write $\left(a_{1} \cdots a_{s} \mid b_{1} \cdots b_{t}\right)$ for $\left.\sum_{i=1}^{s} a_{i}+\sum_{j=1}^{t} b_{j}\right)$.

$$
\begin{gathered}
\left(\left.4\right|_{-}\right) \stackrel{\delta}{\longrightarrow}\left({ }_{-} \mid 31\right) \\
\left(\_\mid 4\right) \stackrel{\xi}{\longrightarrow}(1 \mid 3) \\
\left(\left.31\right|_{-}\right) \stackrel{\delta}{\longrightarrow}(1 \mid 21) \\
(3 \mid 1) \stackrel{\delta}{\longrightarrow}(-\mid 211) \\
(21 \mid 1) \stackrel{\delta}{\longrightarrow}(1 \mid 111) \\
(2 \mid 11) \stackrel{\delta}{\longrightarrow}(-\mid 1111) .
\end{gathered}
$$

The partitions $(2 \mid 2)$ and $(-\mid 22)$ are unpaired.

3. Generalizations of (1.1), (1.3), and (1.4). We begin by remarking that in Franklin's proof of the Euler pentagonal number theorem, the sum of the number of parts plus the largest part of the partition is unaltered by the transformations applied to the Ferrars graph of the partition. to be

Definition [3; p. 135]. We define the $(a, b)$-rank of a partition $\pi$

$$
[a \cdot l(\pi)]-[b \cdot \#(\pi)],
$$

where $l(\pi)$ is the largest part of $\pi, \sharp(\pi)$ is the number of parts of $\pi$, and $[x]$ is the greatest integer function.

Thus the $(1,-1)$-rank is invariant under Franklin's transformations. Let $Q_{e}(r, n)$ (resp. $Q_{0}(r, n)$ ) denote the number of partitions of $n$ into an even (resp. odd) number of distinct parts with $(1,-1)$ rank equal to $r$. Then 


$$
\begin{gathered}
1-\sum_{n=1}^{\infty}(y q)_{n-1} y^{n+1} q^{n} \\
=\sum_{n=0}^{\infty} \frac{(-1)^{n} y^{2 n} q^{1 / 2 n(n+1)}}{(-y q)_{n}} \\
=1+\sum_{n=1}^{\infty} \sum_{r=1}^{\infty}\left(Q_{e}(r, n)-Q_{0}(r, n)\right) y^{r} q^{n} .
\end{gathered}
$$

Franklin's proof tells us that the above expressions also equal

$$
1+\sum_{n=1}^{\infty}(-1)^{n}\left(q^{1 / 2(n)(3 n-1)} y^{3 n-1}+q^{1 / 2(n)(3 n+1)} y^{3 n}\right) \cdot
$$

This observation is discussed in detail by Subbarao [17].

This series of identities is well-known; L. J. Rogers [11; p. 333] observed that the series in (3.2) and (3.4) are equal, and N. J. Fine [5], [6] has proved that all these series are equal; however, all these proofs are analytic.

The same sort of generalization may be obtained from our proofs of Theorems 1 and 2. Taking Theorem 1 as an example, we first remark that if

$$
\frac{t q^{2 n+1}\left(1+t q^{2}\right)\left(1+t q^{4}\right) \cdots\left(1+t q^{2 n}\right)}{(1-t q)\left(1-t q^{3}\right) \cdots\left(1-t q^{2 n+1}\right)}=\sum_{k=0}^{\infty} \sum_{m=0}^{\infty} a_{m, k}(2 n+1) t^{m} q^{k}
$$

and

$$
\frac{t q^{2 n+2}\left(1+t q^{2}\right)\left(1+t q^{4}\right) \cdots\left(1+t q^{2 n}\right)}{(1-t q)\left(1-t q^{3}\right) \cdots\left(1-t q^{2 n+1}\right)}=\sum_{k=0}^{\infty} \sum_{m=0}^{\infty} a_{m, k}(2 n+2) t^{m} q^{k}
$$

then clearly $a_{m, k}(n)$ is the number of partitions of $k$ into $m$ parts in which no even part is repeated and the largest part is $n$. Hence

$$
\begin{aligned}
1-\sum_{n=0}^{\infty} & t^{n+1} q^{2 n+1}\left(t q^{2} ; q^{2}\right) n /\left(-t q ; q^{2}\right)_{n+1}-\sum_{n=0}^{\infty} t^{n+2} q^{2 n+2}\left(t q^{2} ; q^{2}\right)_{n} /\left(-t q ; q^{2}\right)_{n+1} \\
= & 1+\sum_{n=0}^{\infty} t^{n} \sum_{k=0}^{\infty} \sum_{m=0}^{\infty} a_{m, k}(2 n+1)(-t)^{m} q^{k} \\
& +\sum_{n=0}^{\infty} t^{n+1} \sum_{k=0}^{\infty} \sum_{m=0}^{\infty} a_{m, k}(2 n+2)(-t)^{m} q^{k} \\
= & 1+\sum_{k=0}^{\infty} \sum_{m=0}^{\infty} t^{m} q^{k}\left(\sum_{n=0}^{\infty} a_{m-n, k}(2 n+1)(-1)^{m-n}\right. \\
& \left.+\sum_{n=0}^{\infty} a_{m-n-1, k}(2 n+2)(-1)^{m-n-1}\right) \\
= & 1+\sum_{k=0}^{\infty} \sum_{m=0}^{\infty} t^{m} q^{k} b_{m, k} .
\end{aligned}
$$

Now $a_{m-n, k}(2 n+1)$ is the number of partitions of $k$ with $2 n+1$ as largest part and $(1 / 2,-1)$-rank equal to $m$; while $a_{m-n-1}(2 n+2)$ is 
the number of partitions of $k$ with $2 n+2$ as largest part and $(1 / 2,-1)$ rank equal to $m$. Therefore $b_{m, k}$ counts the excess of partitions of $k$ with $(1 / 2,-1)$-rank $m$ and an even number of parts over those with an odd number of parts. Since the $(1 / 2,-1)$-rank is invariant under the transformations listed in Theorem 1 while parity of the number of parts is always altered, we see that

$$
b_{m, k}= \begin{cases}(-1)^{\nu} & \text { if } m=2 \nu-1, k=2 \nu^{2}-\nu \\ (-1)^{\nu} & \text { if } m=2 \nu, k=2 \nu^{2}+\nu \\ 0 & \text { otherwise } .\end{cases}
$$

Hence

$$
\begin{aligned}
1- & \sum_{n=0}^{\infty} t^{n+1} q^{2 n+1}\left(t q^{2} ; q^{2}\right)_{n} /\left(-t q ; q^{2}\right)_{n+1} \\
- & \sum_{n=0}^{\infty} t^{n+2} q^{2 n+2}\left(t q^{2} ; q^{2}\right)_{n} /\left(-t q ; q^{2}\right)_{n+1} \\
& =1+\sum_{n=1}^{\infty}(-1)^{n} t^{2 n-1} q^{2 n^{2}-n}\left(1+t q^{2 n}\right) .
\end{aligned}
$$

In Theorem 2, the $(1,-1)$-rank is invariant under all transformations. Hence using an argument like that used to prove (3.5), we may deduce that

$$
1-2 \sum_{n=1}^{\infty} \frac{(t q)_{n-1} t^{n+1} q^{n}}{(-t q)_{n}}=1-2 \sum_{n=1}^{\infty} t^{2 n}(-1)^{n} q^{n^{2}} .
$$

In searching the literature, we find that (3.5) and (3.6) as well as our generalization Franklin's result are special cases of the RogersFine identity (1.6).

We remark that (1.6) is a special case of a general theorem due to Watson [12; p. 100, eq. (3.4. 1.5)]

$$
\begin{aligned}
\sum_{n=0}^{\infty} & \frac{(a)_{n}(b)_{n}(c)_{n}(d)_{n}(e)_{n}(f)_{n}\left(1-a q^{2 n}\right)\left(a^{2} q^{2} / b c d e f\right)^{n}}{(q)_{n}(a q / b)_{n}(a q / c)_{n}(a q / d)_{n}(a q / e)_{n}(a q / f)_{n}(1-a)} \\
= & \frac{(a q)_{\infty}(a q / e f)_{\infty}(a q / d f)_{\infty}(a q / d e)_{\infty}}{(a q / d)_{\infty}(a q / e)_{\infty}(a q / f)_{\infty}(a q / d e f)_{\infty}} \\
& \times \sum_{n=0}^{\infty} \frac{(a q / b c)_{n}(d)_{n}(e)_{n}(f)_{n} q^{n}}{(q)_{n}(d e f / a)_{n}(a q / b)_{n}(a q / c)_{n}}
\end{aligned}
$$

where $f=q^{-N}$ ( $N$ a positive integer).

In (3.7), let $N \rightarrow \infty$ (i.e. $f \rightarrow \infty$ ), then let $c \rightarrow \infty$ and set $d=q$. Thus

$$
\sum_{n=0}^{\infty} \frac{\left(1-a q^{2 n}\right)(b)_{n}(e)_{n} q^{n^{2}} a^{2 n}(b e)^{-n}}{(1-a)(a q / b)_{n}(a q / e)_{n}}=\frac{\left(1-\frac{a}{e}\right)}{1-a} \sum_{n=0}^{\infty} \frac{(e)_{n}\left(\frac{a}{e}\right)^{n}}{\left(\frac{a q}{b}\right)_{n}}
$$


This is (1.6) written with different variables. In the next section, we shall give an arithmetic proof of the complete Rogers-Fine identity. As will become evident, the proof will markedly differ from the proofs of Theorems 1 and 2 .

4. Arithmetic proof of (1.6). First we make some simple changes of variables in (1.6): replace $q$ by $q^{2}$, then replace $a$ by $a q$, $b$ by $b q^{2}, t$ by $t q^{2}$ and multiply both sides by $b /\left(1-t q^{2}\right)$. This yields

$$
\begin{aligned}
& \sum_{n=0}^{\infty} \frac{\left(-a q ; q^{2}\right)_{n} b t^{n} q^{2 n}}{\left(b q^{2} ; q^{2}\right)_{n}} \\
& \quad=\sum_{n=0}^{\infty} \frac{\left(-a q ; q^{2}\right)_{n}\left(-a t q^{3} / b ; q^{2}\right)_{n}\left(1+a t q^{4 n+3}\right) b^{n+1} t^{n} q^{2 n^{2}+2 n}}{\left(b q^{2} ; q^{2}\right)_{n}\left(t q^{2} ; q^{2}\right)_{n+1}} .
\end{aligned}
$$

The coefficient of $a^{k} b^{l} t^{m} q^{n}$ on the left-hand side of (4.1) is $R(k, l, m, n)$ the number of partitions of $n$ with no repeated odd parts, where there are $l$ even parts, $k$ odd parts, and the largest part is $2 m$.

To obtain the right-hand side of (4.1), we form the Ferrars graph of such partitions and dissect them as follows:

Instead of looking at the Durfee square, we consider: a modified Durfee rectangle which consists of the largest possible rectangle of nodes with sides $2 N$ horizontally by $N+1$ vertically. For example the following illustrates the rectangle related to the partition $68=$ $12+10+10+10+9+8+4+4+1$ (neglect the small dotted circle and square, they are for future reference):

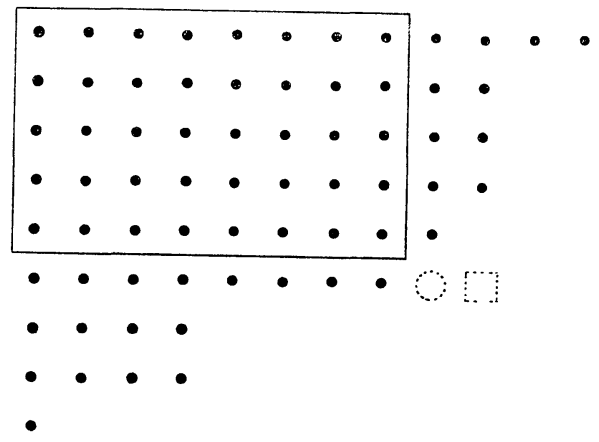

Figure 1

In this case $N=4$, and the rectangle is 8 by 5 . We remark that if there were a node in the small dotted square (and therefore also in the small dotted circle), then there would exist a larger rectangle of the required sort. Hence after the modified Durfee retangle is determined, the position one down and two to the right will not contain a node.

We now divide our considerations into two cases. 
I. The general position indicated by the dotted circle does not contain a node. The portion of the partition below the modified Durfee retangle has a generating function:

$$
\frac{(1+a q) \cdots\left(1+a q^{2 N-1}\right)}{\left(1-b q^{2}\right) \cdots\left(1-b q^{2 N}\right)}=\frac{\left(-a q ; q^{2}\right)_{N}}{\left(b q^{2} ; q^{2}\right)_{N}} .
$$

Now $b^{N+1} t^{N} q^{2 N^{2}+2 N}$ is the generating function related to the portion of the partition contained in the modified Durfee rectangle. Note that the $t^{N}$ correctly counts $1 / 2$ the contribution of the top of the rectangle to the largest part of the partition. Also with the factor $b^{N+1}$ we must remember to delete from this exponent the number of odd parts appearing to the right of the modified Durfee rectangle.

Finally (for case I) we note that

$$
\frac{\left(1+\frac{a t q^{3}}{b}\right) \cdots\left(1+\frac{a t q^{2 N+1}}{b}\right)}{\left(1-t q^{2}\right) \cdots\left(1-t q^{2 N+2}\right)}=\frac{\left(-\frac{a t q^{3}}{b} ; q^{2}\right)_{N}}{\left(t q^{2} ; q^{2}\right)_{N+1}}
$$

is the generating function for the portion of the partition to the right of our rectangle provided we interpret the partitions generated as follows:

We initially think of the above function as generating partitions with parts $\geqq 2$, with distinct odd parts, and with each part $\geqq 2 N+2$. We transform such partitions by representing each even part $2 \nu$ as two columns of $\nu$ nodes each and each odd part $2 \mu+1$ as two columns of $\mu+1$ and $\mu$ nodes respectively. The exponent of $t$ will be $1 / 2$ the number of nodes along the top row, and there will be an even number of nodes along the top row (since originally 1 could not appear). The exponent of $b$ is the negative of the number of odd parts, which (recalling our previous comment on the exponent of $b$ ) is just what we want, and the exponent of $a$ still counts the number of rows with an odd number of parts. Consequently the generating function $R(k, l, m, n)$ with modified Durfee rectangle measuring $2 N \times(N+1)$ in Case $I$ is just

$$
\frac{\left(-a q ; q^{2}\right)_{N}\left(-\frac{a t q^{3}}{b} ; q^{2}\right)_{N} b^{N+1} t^{N} q^{2 N^{2}+2 N}}{\left(b q^{2} ; q^{2}\right)_{N}\left(t q^{2} ; q^{2}\right)_{N+1}} .
$$

II. The general position indicated by the dotted circle does contain a node. In this case the position just above the dotted square must contain a node or else the odd part $2 N+1$ would be repeated, a prescribed condition. Also the position directly below the dotted circle must not contain a node for the same reason.

The argument now proceeds exactly as in Case I except that the 
contribution from the modified Durfee rectangle is now extended to include the row below the rectangle as well as the two columns to the right of the rectangle. Thus the generating function for this portion of the partition is

$$
a b^{N+1} t^{N+1} q^{(2 N+2)(N+1)-1}=a b^{N+1} t^{N+1} q^{2 N^{2}+6 N+3} .
$$

Hence the total generating function in Case II is just

$$
\frac{\left(-a q ; q^{2}\right)_{N}\left(-\frac{a t q^{3}}{b} ; q^{2}\right)_{N} a b^{N+1} t^{N+1} q^{2 N^{2}+6 N+3}}{\left(b q^{2} ; q^{2}\right)_{N}\left(t q^{2} ; q^{2}\right)_{N+1}} .
$$

Adding the generating functions obtained from Cases I and II we obtain in Nth term in the series on the right-hand side of (4.1). Since this is the generating function for partitions of the type enumerated by $R(k, l, m, n)$ with modified Durfee rectangle measuring $2 N X(N+1)$, we see that the sum running from $N=0$ to $\infty$ produces the generating function for $R(k, l, m, n)$. Hence since the left-hand side of (4.1) has already been seen to be the generating function for $R(k, l, m, n)$, we see that (4.1) and so also (1.6) are established.

The final section of this paper is devoted to other basic hypergeometric series identities that are derivable from considerations of partitions without repeated odd parts.

5. Conclusion. The study of partitions in which no odd parts are repeated can be utilized to prove two further results of interest. First we consider

$$
\sum_{n=0}^{\infty} \frac{\left(-a q^{3} ; q^{2}\right)_{n} b t^{n+1} q^{2 n+2}}{\left(b q^{2} ; q^{2}\right)_{n+1}}=\sum_{n=0}^{\infty} \frac{\left(-\frac{a t q^{3}}{b} ; q^{2}\right)_{n} t b^{n+1} q^{2 n+2}}{\left(t q^{2} ; q^{2}\right)_{n+1}} .
$$

This result was first stated and proved by N. J. Fine [6]; it is a special case of Heine's fundamental transformation (set $\beta=q$ in equation (I1) of [1] to obtain an identity equivalent to (5.1)).

The coefficient of $a^{k} b^{l} t^{m} q^{n}$ on the left-hand side of (5.1) is say $S(k, l, m, n)$, the number of partitions of $n$ into parts each larger than 1 , with no repeated odd parts, with largest part $2 m$, with $l$ even parts and $k$ odd parts.

Now we form a modified Ferrars graph for partitions of the type enumerated by $S(k, l, m, n)$.

Each even part $2 j$ is to be represented as two rows of $j$ nodes, and each odd part $2 h+1$ is to be represented as two rows of $h+1$ and $h$ nodes respectively.

We now consider the ordinary conjugate partition formed from 
our representation. Since originally the largest part was $2 m$, there are now $m$ columns, and therefore $m$ parts in this conjugate partition. Since there were $k$ odd distinct parts originally, there are still $k$ odd distinct parts in this conjugate partition. Since 1 did not appear originally, the largest part in this conjugate partition is $2 k+$ $2 l$. Since originally the largest part was even (namely $2 \mathrm{~m}$ ), 1 will not appear in this conjugate. Thus this conjugate is a partition of the type enumerated by $S(k, m-k, k+l, n)$. Since the preceding transformation is clearly reversible, we see that

$$
S(k, l, m, n)=S(k, m-k, k+l, n) \text {. }
$$

Thus if $f(a, b, t, q)$ denotes the left hand side of (5.1), then by (5.2)

$$
f(a, b, t, q)=f(a t / b, t, b, q) .
$$

This last identity is precisely (5.1).

Finally we consider another identity due to Fine [6]:

$$
\sum_{n=0}^{\infty} \frac{\left(-a q ; q^{2}\right)_{n} b t^{n} q^{2 n}}{\left(b q^{2} ; q^{2}\right)_{n}}=\sum_{n=0}^{\infty} \frac{\left(-b q / a ; q^{2}\right)_{n} b a^{n} t^{n} q^{n^{2}+2 n}}{\left(b q^{2}: q^{2}\right)_{n}\left(t q^{2} ; q^{2}\right)_{n+1}} \text {. }
$$

By setting $b=1$ in (5.4) and utilizing a summation due to Heine [12; p. 92, eq. (3.2.2.11)], we obtain a well-known identity of Cauchy:

$$
\begin{aligned}
\sum_{n=0}^{\infty} \frac{\left(-q / a ; q^{2}\right)_{n} a^{n} t^{n} q^{n^{2}+2 n}}{\left(q^{2} ; q^{2}\right)_{n}\left(t q^{2} ; q^{2}\right)_{n+1}} & =1 \dot{\phi}_{0}\left(-a q, t q^{2}, q^{3}\right) \\
& =\frac{\left(-a t q^{3} ; q^{2}\right)_{\infty}}{\left(t q^{2} ; q^{2}\right)_{\infty}} .
\end{aligned}
$$

Indeed once (5.4) is established arithmetically we shall also have an arithmetric proof of (5.5) by merely combining our proof of (5.4) with the proof of Lemma 2 in [2]. This essentially answers the question posed in [4; Section 3] asking for an arithmetic proof of Cauchy's identity.

The left-hand side of (5.4) is identical with the left-hand side of (4.1) and is therefore the generating function for $R(k, l, m, n)$, the number of partitions of $n$ with no repeated odd parts, $l$ even parts, $k$ odd parts, and $2 m$ as largest part.

We now rewrite the right-hand side of (5.4) in the following form:

$$
\sum_{N=0}^{\infty} \frac{\left(1+\frac{a}{b} q^{-1}\right)\left(1+\frac{a}{b} q^{-3}\right) \cdots\left(1+\frac{a}{b} q^{-2 N+1}\right) b^{N+1} t^{N} q^{2 N(N+1)}}{\left(1-b q^{2}\right)\left(1-b q^{4}\right) \cdots\left(1-b q^{2 N}\right)\left(1-t q^{2}\right)\left(1-t q^{4}\right) \cdots\left(1-t q^{2 N+2}\right)} .
$$

Definition. We shall say that the Cauchy order $\mathscr{C}(\pi)$ of a partition $\pi$ is the largest integer $\nu$ for which the sum of the number of 
odd parts in $\pi$ plus the number of even parts in $\pi$ exceeding $2 \nu-1$ is at least $\nu+1$.

Thus if $\pi$ is given by $78=12+10+10+10+9+8+4+4+1$, then $\mathscr{C}(\pi)=5$ since there are 2 odd parts in $\pi$ and 4 even parts exceeding $9,2+4=5+1$, and 5 is the largest $\nu$ possible.

We remark that $\mathscr{C}(\pi)$ always exists because if $c(\pi: \nu)$ denotes (the number odd parts of $\pi$ ) plus (the number of even parts of $\pi$ exceeding $2 \nu-1$ ) minus $(\nu+1)$, then $c(\pi: 0) \geqq 0$ (for all nonempty partitions) and $c(\pi: M)<0$ for $M$ sufficiently large.

To conclude our proof we need only show that the $N$-th summand of the series in (5.6) is the generating function for partitions enumerated by $R(k, l, m, n)$ with Cauchy order equal to $N$.

First we note that $\left(b q^{2} ; q^{2}\right)_{N}^{-1}$ is the generating function for partitions into even parts each not exceeding $2 N$, and $b^{N+1} t^{N} q^{2 N(N+1)} /\left(t q^{2} ; q^{2}\right)_{N+1}$ is the generating function for partitions into $N+1$ even parts each greater than or equal to $2 N$ where the exponent of $t$ is half the largest part. It remains to show that the product of these two generating functions with

$$
\left(1+\frac{a}{b} q^{-1}\right)\left(1+\frac{a}{b} q^{-3}\right) \cdots\left(1+\frac{a}{b} q^{-2 N+1}\right)
$$

yields the desired generating function related to Cauchy order $N$.

In the simplest possible situation for determining the Cauchy order, there would be no odd parts; in this situation we see that the product of the first two generating functions mentioned in the preceding paragraph generates such partitions with Cauchy order $N$. Furthermore we note that if in a partition such as has just been described we replace some of the $N+1$ even parts larger than $2 N-1$ by odd parts, the Cauchy order will not be altered.

Now let us now note that one obtains a general partition $\pi$ of the type enumerated by $R(k, l, m, n)$ with $\mathscr{C}(\pi)=N$ by starting with such a partition that contains no odd parts and subtracting $2\left(j_{i}+i-1\right)-$ 1 from the $\left(j_{i}+i-1\right)$-st part, where $i$ runs from 1 to $k$ and $j_{i}$ is a nondecreasing sequence with $1 \leqq j_{i} \leqq \nu+1-k$. That such a subtraction yields a positive odd part follows from the fact that the original Cauchy order was $N$. Note that

$$
1 \leqq 2\left(j_{i}+i-1\right)-1 \leqq 2 N-1 \text {. }
$$

Conversely given any set of distinct odd numbers lying in [1, 2N-1] it is clear how to determine the related sequence $j_{i}$. The Cauchy order is clearly unaltered as we have observed previously.

Thus to obtain the generating function for partitions of the type enumerated by $R(k, l, m, n)$ subject to the condition that $\mathscr{C}(\pi)=N$, 
we take the generating function for such partitions with no odd parts, namely

$$
\frac{b^{N+1} t^{N} q^{2 N(N+1)}}{\left(b q^{2} ; q^{2}\right)_{N}\left(t q^{2} ; q^{2}\right)_{N+1}}
$$

and we multiply by the generating function which will subtract off distinct odd parts in the range [1, $2 N-1]$, namely

$$
\left(1+\frac{a}{b} q^{-1}\right)\left(1+\frac{a}{b} q^{-3}\right) \cdots\left(1+\frac{a}{b} q^{-2 N+1}\right) .
$$

Hence we have that the $N$ th term in (5.6) is the required generating function related to Cauchy order $N$. Summing over all Cauchy orders, we see that the series in (5.6) equals the generating function $R(k, l, m, n)$. Thus (5.4) is proved.

\section{REFERENCES}

1. G. E. Andrews, q-Identities of Auluck, Carlitz, and Rogers, Duke Math. J., 33 (1966), 575-582.

2. - On a calculus of partition functions, Pacific J. Math., 31 (1969), 555562.

3. - On basic hypergeometric series, mock-theta functions, and partitions (II), Quart. J. Math. (2), 17 (1966), 132-143.

4. G. E. Andrews, M. V. Subbarao, and M. Vidyasagar, A family of combinatorial identities, Canad. Math. Bull., 15 (1972), 11-18.

5. N. J. Fine, Some new results on partitions, Proc. Nat. Acad. Sci., 34 (1948), 616618.

6. Some Basic Hypergeometric Series and Applications, (to appear).

7. F. Franklin, Sur le development du produit infini $(1-x)\left(1-x^{2}\right)\left(1-x^{3}\right)\left(1-x^{4}\right) \ldots$, Comptes Rendus de l'Acad. des Sciences (Paris), 92 (1881), 448-450.

8. C. F. Gauss, Werke, Bd. 3, Koniglichen Gesellschaft der Wissenschaften, Gottingen, 1866.

9. R. K. Guy, A theorem in partitions, Univ. of Calgary, Research paper No. 11, 1967.

10. G. H. Hardy and E. M. Wright, An Introduction to the Theory of Numbers, 4th ed., Oxford University Press, Oxford, 1960.

11. L. J. Rogers, On two theorems of combinatory analysis and some allied identities, Proc. London Math. Soc., (2), 16 (1917), 315-336.

12. L. J. Slater Generalized Hypergeometric Functions, Cambridge University Press, Cambridge, 1966.

13. C. Sudler, Two enumerative proofs of an identity of Jacobi, Proc. Edinburgh Math. Soc., 15 (1966), 67-71.

14. J. J. Sylvester, A constructive theory of partitions..., Collected Papers, Vol. 4, Cambridge University Press, Cambridge, 1912.

15. K. T. Vahlen, Beiträge zu einer additiven Zahlentheorie, J. fur die reine und angew Math., 112 (1893), 1-36.

16. E. M. Wright, An enumerative proof of an identity of Jacobi, J. London Math. Soc., 40 (1965), 55-57.

17. M. V. Subbarao, Combinatorial proofs of some identities, Proc. of the Washington State Number Theory Conference, Pullman, 1971. 
Received March 22, 1971 and in revised form May 31, 1971. Partially supported by National Science Foundation Grant GP-9660.

The Pennsylvania State University

AND

Massachusetts Institute of Technology 


\title{
PACIFIC JOURNAL OF MATHEMATICS
}

\author{
EDITORS
}

H. SAMELSON

Stanford University

Stanford, California 94305

C. R. HOBBY

University of Washington

Seattle, Washington 98105

\section{J. DugundJI}

Department of Mathematics

University of Southern California

Los Angeles, California 90007

RICHARD ARENS

University of California

Los Angeles, California 90024

\section{ASSOCIATE EDITORS}
E. F. BeCKeNBACH
B. H. NeUmanN
F. WoLF
K. YoSHIDA

\section{SUPPORTING INSTITUTIONS}

\author{
UNIVERSITY OF BRITISH COLUMBIA \\ CALIFORNIA INSTITUTE OF TECHNOLOGY \\ UNIVERSITY OF CALIFORNIA \\ MONTANA STATE UNIVERSITY \\ UNIVERSITY OF NEVADA \\ NEW MEXICO STATE UNIVERSITY \\ OREGON STATE UNIVERSITY \\ UNIVERSITY OF OREGON \\ OSAKA UNIVERSITY
}

\author{
UNIVERSITY OF SOUTHERN CALIFORNIA \\ STANFORD UNIVERSITY \\ UNIVERSITY OF TOKYO \\ UNIVERSITY OF UTAH \\ WASHINGTON STATE UNIVERSITY \\ UNIVERSITY OF WASHINGTON

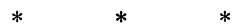 \\ AMERICAN MATHEMATICAL SOCIETY \\ NAVAL WEAPONS CENTER
}

The Supporting Institutions listed above contribute to the cost of publication of this Journal, but they are not owners or publishers and have no responsibility for its content or policies.

Mathematical papers intended for publication in the Pacific Journal of Mathematics should be in typed form or offset-reproduced, (not dittoed), double spaced with large margins. Underline Greek letters in red, German in green, and script in blue. The first paragraph or two must be capable of being used separately as a synopsis of the entire paper. The editorial "we" must not be used in the synopsis, and items of the bibliography should not be cited there unless absolutely necessary, in which case they must be identified by author and Journal, rather than by item number. Manuscripts, in duplicate if possible, may be sent to any one of the four editors. Please classify according to the scheme of Math. Rev. Index to Vol. 39. All other communications to the editors should be addressed to the managing editor, Richard Arens, University of California, Los Angeles, California, 90024.

50 reprints are provided free for each article; additional copies may be obtained at cost in multiples of 50 .

The Pacific Journal of Mathematics is published monthly. Effective with Volume 16 the price per volume (3 numbers) is $\$ 8.00$; single issues, $\$ 3.00$. Special price for current issues to individual faculty members of supporting institutions and to individual members of the American Mathematical Society: $\$ 4.00$ per volume; single issues $\$ 1.50$. Back numbers are available.

Subscriptions, orders for back numbers, and changes of address should be sent to Pacific Journal of Mathematics, 103 Highland Boulevard, Berkeley, California, 94708.

PUBLISHED BY PACIFIC JOURNAL OF MATHEMATICS, A NON-PROFIT CORPORATION

Printed at Kokusai Bunken Insatsusha (International Academic Printing Co., Ltd.), 270, 3-chome Totsuka-cho, Shinjuku-ku, Tokyo 160, Japan. 


\section{Pacific Journal of Mathematics}

\section{Vol. 41, No. $3 \quad$ BadMonth, 1972}

George E. Andrews, Two theorems of Gauss and allied identities proved arithmetically.................................. 563

Stefan Bergman, On pseudo-conformal mappings of circular domains . . . . 579

Beverly L. Brechner, On the non-monotony of dimension ............ 587

Richard Anthony Brualdi and John H. Mason, Transversal matroids and Hall's theorem .................................... 601

Philip Throop Church and James Timourian, Differentiable maps with

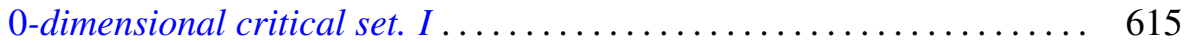

John H. E. Cohn, Squares in some recurrent sequences ............. 631

Robert S. Cunningham, Edgar Andrews Rutter and Darrell R. Turnidge, Rings of quotients of endomorphism rings of projective modules ......

Eldon Dyer and S. Eilenberg, An adjunction theorem for locally equiconnected spaces................................... 669

Michael W. Evans, On commutative P. P. rings................. 687

Ronald Lewis Graham, Hans Sylvain Witsenhausen and Hans Zassenhaus, On tightest packings in the Minkowski plane ..................

Stanley P. Gudder, Partial algebraic structures associated with

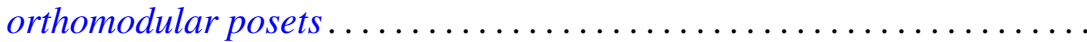

Karl Edwin Gustafson and Gunter Lumer, Multiplicative perturbation of

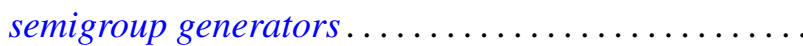

Kurt Kreith and Curtis Clyde Travis, Jr., Oscillation criteria for selfadjoint elliptic equations...

Lawrence Louis Larmore, Twisted cohomology theories and the single obstruction to lifting...........................

Jorge Martinez, Tensor products of partially ordered groups . . .

Robert Alan Morris, The inflation-restriction theorem for Amitsur cohomology ...

Leo Sario and Cecilia Wang, The class of $(p, q)$-biharmonic functions ...

Manda Butchi Suryanarayana, On multidimensional integral equations of

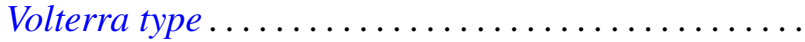

